\title{
Comportamiento de compra en el comercio móvil: la importancia de los rasgos de personalidad
}

\author{
Zaira Camoiras-Rodríguez / Concepción Varela-Neira \\ Universidade de Santiago de Compostela - Facultade de Ciencias Económicas e Empresariais
}

Recibido: 28 de abril de 2020 / Aceptado: 5 de noviembre de 2020

\begin{abstract}
Resumen
Las ventajas que el comercio móvil proporciona han atraído la atención de empresas y consumidores. A pesar de sus potenciales beneficios, la investigación sobre los factores que influyen en su frecuencia de uso y compra es todavía escasa. Este estudio contribuye a alcanzar un mayor conocimiento y comprensión sobre los factores que influyen en la frecuencia de compra en el comercio móvil al vincular el Modelo de Aceptación de Tecnología (TAM) con la literatura de los rasgos de personalidad. Esta investigación emplea una muestra de 200 individuos que poseen dispositivos móviles con acceso a Internet. La técnica utilizada para contrastar las hipótesis es el análisis path. Los resultados muestran el efecto indirecto de la necesidad de recursos materiales y de la orientación a la tarea sobre la frecuencia de compra en el comercio móvil. Además, los resultados reflejan el importante papel mediador que tiene la tendencia a la compra impulsiva en el nivel de frecuencia de compra.
\end{abstract}

\section{Palabras clave}

Rasgos de personalidad / Necesidad de recursos materiales / Orientación a la tarea / Tendencia a la compra impulsiva / Comercio móvil.

\section{Mobile commerce purchase behaviour: The importance of personality traits}

\begin{abstract}
The advantages that mobile commerce provides have attracted the attention of companies and consumers. Despite its potential benefits, research on the factors that influence how often it is used and bought is still scarce. This research contributes to a greater knowledge and understanding of the factors that influence how often it is bought in a mobile environment by relating the Technology Acceptance Model (TAM) to personality traits literature. This research is based on a sample of 200 individuals who have mobile devices with Internet access. The technique used to test the hypotheses is path analysis. The results show the indirect effect of the need for material resources and task orientation on the number of purchases in mobile commerce. Furthermore, the results reflect the important mediating role of an impulsive purchasing tendency in the level of purchase frequency.
\end{abstract}

\section{Keywords}

Personality traits / Need for material resources / Task orientation / Impulsive buying tendency / Mobile commerce..

JEL Codes: M31.

\section{Introducción}

Los avances tecnológicos acontecidos en los últimos años están creando un medio cada vez más competitivo (Gupta y Arora, 2017). En concreto, el crecimiento en el uso de dispositivos móviles, especialmente smartphones, supone un importante desarrollo para múltiples sectores comerciales, y en especial para el sector minorista (Alalwan et al., 2020; Kapoor y Vij, 2018). Así, este incremento ha contribuido a la emergencia y desarrollo del comercio móvil (m-commerce), que consiste en un conjunto

\footnotetext{
* Correspondencia autora: zaira.camoiras@usc.es
} 
de actividades que se realizan a través de dispositivos móviles, como smartphones, portátiles o tabletas (Zhang, Zhu y Liu, 2012), que facilitan el uso y permiten el aprovechamiento de las ventajas que estos aparatos proporcionan en materia de disponibilidad geográfica y temporal (Kim, Li y Kim, 2015).

Los beneficios que permite obtener el comercio móvil están atrayendo la expectación y el interés de una nueva generación de consumidores (Gupta y Arora, 2017), transformado por completo sus experiencias de compra (Chopdar, Korfiatis, Sivakumar y Lytras, 2018; Natarajan, Balasubramanian y Kasilingam, 2018). Este fenómeno puede constatarse en el elevado número de personas que en la última década están empleando sus dispositivos móviles para realizar esta acción (Patel, Das, Chatterjee y Shukla, 2020). En concreto, en España el 63\% de los compradores online han realizado compras, al menos una vez, desde sus dispositivos móviles en el año 2018 (Statista, 2020), aumentando la cifra de compradores móviles en los últimos años a un ritmo superior a la media de los países de Europa occidental (Kats, 2020).

El comercio móvil no solo ha atraído la atención de los consumidores sino también de investigadores y empresas (Ashraf, Thongpapanl, Menguc y Northey, 2017; Huang y Zhou, 2018; Kim et al., 2015; Kim, Kim, Choi y Trivedi, 2017), dado su potencial impacto en los negocios y en la industria en general (Chong, 2013). Los resultados publicados por Kats (2020) muestran que en el año 2019 las ventas de comercio móvil minorista en España crecieron un 21,7\%, alcanzando un valor de 10,24 mil millones de dólares, y las previsiones para 2020 reflejan un crecimiento del 17,8\%, lo que supondría unas ventas de 12,06 mil millones de dólares. Como consecuencia, la industria minorista, al reconocer el gran potencial de la tecnología móvil, ofrece cada vez con más frecuencia compras móviles, con el objetivo de proporcionar ofertas más específicas a sus clientes (Chopdar et al., 2018) y lograr una interacción más estrecha con ellos (Natarajan et al., 2018). A pesar de los potenciales beneficios del comercio móvil, la investigación acerca de los factores que influyen en su adopción y frecuencia de uso es todavía escasa (Zhang et al., 2012), centrándose la mayor parte de ella, además, en la adopción de esta tecnología (Jiménez, San-Martín y Puente, 2019) y no en sus niveles de uso. Este trabajo trata de ayudar en este campo, al estudiar los antecedentes de la frecuencia con la que los consumidores compran a través de dispositivos móviles.

La mayoría de los estudios realizados sobre el comercio móvil están basados en el modelo TAM (por ejemplo, Bruner y Kumar, 2005; Chen y Lan, 2018; Faqih y Jaradat, 2015; Jiménez et al., 2019; Luarn y Lin, 2005; Natarajan et al., 2017, 2018; Vahdat, Alizadeh, Quach y Hamelin, 2020; Wu y Wang, 2005), al que le han ido añadiendo diversas variables con el fin de extender y completar el modelo. Este modelo, propuesto por Davis (1989), se centra fundamentalmente en las percepciones internas de los consumidores, y establece que la decisión de utilizar una tecnología viene determinada por la facilidad de uso y por la utilidad percibida de la tecnología en cuestión. No obstante, diversos autores (Faqih y Jaradat, 2015; Jiménez et al., 2019; Okazaki y Méndez, 2013; Vahdat et al., 2020; Venkatesh y Bala, 2008; Zhang et al., 2012) defienden la posibilidad de modificar el modelo TAM a través del uso de diferentes factores o teorías con los que se obtienen, igualmente, resultados válidos y robustos.

En esta línea, la presente investigación propone fusionar el modelo TAM con la literatura de rasgos de personalidad, con el fin de alcanzar un estudio más completo de la frecuencia de compra en el comercio móvil a través de las características individuales de los consumidores. Investigaciones en psicología han destacado los importantes efectos que los rasgos de personalidad tienen en el comportamiento de los individuos (Costa y McCrae, 1992), así como su idoneidad para ser utilizadas como variables antecedente (Otero-López y Villardefrancos, 2013; Soutter, Bates y Mõttus, 2020; Wu, Wang, Lee, Lin y Guo, 2019).

Varios trabajos previos han recalcado la influencia directa e indirecta que los rasgos de personalidad pueden tener en la predicción de tendencias a participar en diversos comportamientos (Al-Samarraie, Eldenfria y Dawoud, 2017; Yazdanparas y Alhenawi, 2017). Siguiendo esta vía de análisis, diversos autores han destacado la necesidad de incluir los rasgos de personalidad en el estudio de la adopción de nuevas tecnologías (Aydin, 2019; Wells, Parboteeah y Valacich, 2011; Wixom y Tood, 2005; Zhou y Lu, 2011). En el ámbito concreto del comercio móvil, algunos autores han comenzado a analizar los efectos de diferentes rasgos de la personalidad en su adopción e intención de uso (Agyei, 
Sun, Abrokwah, Penney y Ofori-Boafo, 2020; Aldás-Manzano, Ruiz-Mafé y Sanz-Blas, 2009; Aydin, 2019; Barnett, Pearson, Pearson y Kellermanns, 2015; Khan, Cao y Pitafi, 2019; Mahatanankoon, 2007; Zhou y Lu, 2011). Sin embargo, y a pesar de su importancia, su estudio es todavía muy limitado (Agyei et al., 2020; Chang, Hsieh y Lin, , 2013; Zhou y Lu, 2011).

En lo que a la personalidad se refiere, esta investigación empleará rasgos de personalidad que la literatura previa ha considerado relevantes para explicar el comportamiento de compra online (Sun y Wu, 2011). Así, este estudio centrará su atención, por un lado, en la necesidad de recursos materiales, que hace referencia al impulso por recoger y poseer bienes tangibles (Mowen, 2000), así como, por otro, en la orientación a la tarea, definida como la disposición duradera de un individuo a establecer objetivos de tareas y a lograr altos niveles de rendimiento en su realización (Mowen, 2000).

Por otro lado, también se incluye la tendencia a la compra impulsiva, que tiene que ver con un rasgo relativamente duradero de un individuo a comprar de forma espontánea e inmediata, sin reflexionar (Rook y Fisher, 1995). Las características y beneficios que permite el comercio móvil están dando lugar a un incremento en la importancia de la compra impulsiva en estos entornos (Turkyilmaz, Erdem y Uslu, 2015; Zheng, Men, Yang y Gong, 2019). No obstante, a pesar de las crecientes evidencias que muestran que el comercio móvil incrementará el comportamiento de compra impulsivo de los consumidores, su estudio es todavía escaso en ámbitos de compra online y móvil (Sun y Wu, 2011; Zheng et al., 2019). La tendencia a la compra impulsiva difiere del comportamiento de compra impulsivo, que viene siendo el resultado de las motivaciones provocadas por esta tendencia (Zhang et al., 2007). De hecho, los consumidores con un impulso a la compra pueden no actuar en consecuencia, ya que algunos factores pueden mediar la relación entre este impulso y su comportamiento. Al mismo tiempo, este rasgo de personalidad se ha relacionado previamente con otros rasgos de personalidad más genéricos (Sun y Wu, 2011; Verplanken y Herabadi, 2001). Por consiguiente, dada la emergente relevancia que la compra impulsiva está experimentando con base en el gran potencial del comercio móvil (Liu y Xiao, 2018), el rasgo de personalidad vinculado a la compra impulsiva se empleará como variable intermediadora en el modelo sobre la frecuencia de compra.

Este estudio trata, por tanto, a través de la combinación de la teoría de aceptación de tecnología con los rasgos de personalidad, de cubrir el gap existente en lo relativo a la escasez de investigaciones que aborden la influencia de la personalidad de los individuos sobre los comportamientos de compra en el comercio móvil (Zhou y Lu, 2011).

\section{Revisión de la literatura y formulación de hipótesis}

\subsection{Tendencia a la compra impulsiva, utilidad percibida y facilidad de uso}

La compra impulsiva hace referencia a la realización de compras de forma imprevista, repentina y no planeada, que se produce en respuesta a estímulos inmediatos y externos (Xiao y Nicholson, 2013), y que suele ir acompañada de sentimientos de emoción y placer (Rook, 1987). Constituyen comportamientos de compra que se producen sin considerar la consistencia de la compra con los objetivos a largo plazo, ideales y planes del individuo que las realiza (Baumeister, 2002).

Los individuos con una tendencia elevada a la compra impulsiva suelen ser irreflexivos en sus pensamientos, dejan en un segundo plano las potenciales consecuencias negativas que sus acciones pueden ocasionar (Kacen y Lee, 2002; Khorrami, Esfidani y Delavari, 2015) y se centran en el sentimiento inmediato de satisfacción que experimentan con las compras (Podoshen y Andrzejewski, 2012; Xiao y Nicholson, 2013). Para los compradores impulsivos, el propio acto de compra provoca un sentimiento inmediato de gratificación (Podoshen y Andrzejewski, 2012; Xiao y Nicholson, 2013). Consecuentemente, existe una mayor probabilidad de que los individuos impulsivos se comporten de manera caprichosa y reaccionen de forma inminente conforme a sus impulsos. La retroalimentación positiva e inmediata que experimentan estos individuos tras realizar la compra impulsiva provoca que el comportamiento de compra realizado tienda a expandirse, tanto en alcance como en frecuencia ( $O^{\prime}$ Guinn y 
Faber, 1989). Este proceso de expansión, denominado "comportamiento de bola de nieve", produce una rotura completa de los esfuerzos de autocontrol del individuo (Baumeister, Heatherton y Tice, 1994). En esta situación, es más probable que los individuos que han realizado compras impulsivas vuelvan a experimentar impulsos de compra con más frecuencia y fuerza que los consumidores que nunca han realizado este tipo de compras, tendiendo a actuar inmediatamente de acuerdo al impulso (Rook y Fisher, 1995). Por tanto, es de esperar que los individuos con elevada tendencia a la compra impulsiva sientan impulsos de compra de manera más recurrente e intensa que otros individuos. Adicionalmente, las personas con tendencia a las compras impulsivas tenderán a conectarse a Internet durante más tiempo y de forma más frecuente en lugares de compra (Lejoyeux, Mathieu, Embouazza, Huet y Lequen, 2007), como puede ser el comercio móvil, lo que incrementará el número de estímulos externos a los que estárán expuestas y facilitará, al mismo tiempo, la posibilidad de que se efectúen las compras, dadas las posibilidades de acceso y disponibilidad que permite el comercio móvil (Kim et al., 2015).

Con base en todo lo expuesto, se propone la siguiente hipótesis:

$-\mathrm{H}_{1}$. La tendencia a la compra impulsiva influye directa y positivamente sobre la frecuencia de compra en el comercio móvil.

Sin embargo, el hecho de experimentar un impulso no siempre implica que el individuo tenga que indefectiblemente ejecutarlo. Existen múltiples factores que pueden actuar como mediadores entre los impulsos experimentados por los individuos a realizar compras impulsivas, y la propia acción de compra desarrollada en el comercio móvil. De acuerdo con lo anterior, sugerimos dos posibles factores de mediación que constituyen percepciones internas de los individuos (Zhang et al., 2012): la facilidad de uso y la utilidad percibida en el comercio móvil.

El modelo TAM constituye una adaptación de la Teoría de Acción Razonada (TRA), la cual explica el comportamiento de los individuos de acuerdo con intenciones y creencias (Fishbein y Ajzen, 1975). El modelo TAM argumenta que ambos, la facilidad de uso y la utilidad percibida para una nueva tecnología, tienen un efecto directo positivo sobre la intención de usar dicha tecnología y, por tanto, su uso (Davis, 1989).

La facilidad de uso se define como el grado en que un individuo considera que la utilización de un determinado sistema está libre de esfuerzo (Davis, 1989). Se relaciona con las características propias de las tecnologías, como simplicidad en la comprensión de contenidos y funciones, así como de aprendizaje y uso (Hernández, Jiménez y Martín, 2009). En general, a medida que aumenta la complejidad, se requiere por parte de los individuos un incremento en el esfuerzo que deben realizar, lo que reducirá la probabilidad de que estos adopten y utilicen una tecnología (Dickerson y Gentry, 1983; Hirschman, 1980). En el caso concreto de esta investigación, esto implica que cuanto más difícil de usar se perciba el comercio móvil, menos uso se hará de él.

La utilidad percibida es el grado en que se considera que la utilización de un sistema determinado por parte de un individuo producirá mejoras en su rendimiento de su trabajo (Davis, 1989). La utilidad percibida afecta al uso de un sistema debido al valor de refuerzo de los resultados (Igbaria y Tan, 1997). Cuando un individuo se da cuenta de que un sistema es útil, considerará que este ofrece una relación uso-rendimiento positiva (Burton-Jones y Hubona, 2006). El hecho de que un sistema sea percibido como útil para lograr determinados objetivos incrementará la probabilidad de uso por parte de los individuos (Igbaria, Guimaraes y Davis, 1995). Así pues, cabe esperar que al aumentar la utilidad percibida del comercio móvil, mayor será el uso que los individuos hagan de él.

Anteriores investigaciones han propuesto y han soportado el efecto de la facilidad de uso (Igbaria, 1992), la utilidad percibida (Igbaria y Toraskar, 1994; Thompson, Higgins y Howell, 1991), y ambos conceptos (Adams, Nelson y Todd, 1992; Roger, 1995; Straub, Limayem y Karahanna-Evaristo, 1995) sobre el uso de un sistema de información. Asimismo, en el caso concreto del comportamiento de compra, se ha encontrado que ambas percepciones influyen de manera directa en el comportamiento de compra actual de los individuos (Lu, Yao y Yu, 2005; Luarn y Lin, 2005), ya que cuanto mayor sea la 
facilidad de uso y la utilidad percibida, mayor será el número de intercambios realizados (Hernandez et al., 2009).

En esta línea, se proponen las siguientes hipótesis:

$-\mathrm{H}_{2}$. La facilidad de uso del comercio móvil tiene una influencia directa y positiva sobre la frecuencia de compra en dicho comercio.

$-\mathrm{H}_{3}$. La utilidad percibida del comercio móvil tiene una influencia directa y positiva sobre la frecuencia de compra en ese comercio.

La impulsividad se ha relacionado con el entusiasmo, con el afán de aventuras y con la actividad, lo que convierte a las personas impulsivas en más predispuestas a asumir riesgos y, en ocasiones, a actuar sin pensar e ignorando hechos concretos al tomar las decisiones (Dickman, 1990). Por otro lado, y dado que para el comprador impulsivo el acto de compra le proporciona un sentimiento inmediato de satisfacción, la compra para ellos es en sí misma una forma de recreación (Silvera, Lavack y Kropp, 2008; Verplanken, Herabadi, Perry y Silvera, 2005). Igualmente, las herramientas disponibles en el comercio móvil que facilitan la navegación y la compra, como pueden ser listas de deseos, búsquedas recientes, sugerencias personalizadas o compra en un clic, ayudan a los compradores impulsivos a satisfacer sus impulsos de manera más rápida y sencilla, lo que provoca que perciban una mayor facilidad de uso en el comercio móvil (Wong, Osman, Jamaluddin y Yin-Fah, 2012). En consecuencia, mantenemos que los consumidores con tendencia a la compra impulsiva percibirán una mayor facilidad de uso del comercio móvil.

Esto nos permite formular la siguiente hipótesis:

$-\mathrm{H}_{4}$. La tendencia a la compra impulsiva tiene una influencia directa y positiva sobre la percepción de facilidad de uso del comercio móvil.

Los consumidores con tendencia a la compra impulsiva percibirán una utilidad adicional en la ubicuidad de consumo que permite el comercio móvil, definida por Cox (2004) como la habilidad para consumir bienes y servicios en cualquier momento y lugar. Esta accesibilidad total e inmediata a las compras que posibilita el comercio móvil (Kim et al., 2015) permitirá a los individuos con tendencia a las compras impulsivas a alcanzar en un menor tiempo la satisfacción consecuente a la adquisición. De igual manera, el ahorro de tiempo y esfuerzo que permite el comercio móvil, dada la posibilidad de realizar las compras en cualquier lugar sin necesidad de desplazarse (Aldás-Manzano et al., 2009), implicará una utilidad extra que será muy valorada por los compradores impulsivos.

Por otra parte, los compradores impulsivos elaboran listas de la compra más abiertas, y son más receptivos a recibir ideas de compra inesperadas que puedan proporcionarles esa satisfacción que desean lo más rápido posible (Khorrami et al., 2015; Rook y Fisher, 1995). Por consiguiente, los individuos que realizan compras impulsivas percibirán una mayor utilidad del comercio móvil gracias a la posibilidad de acceso a información personalizada y a sugerencias adaptadas que este ofrece (Groß, 2015).

De acuerdo con lo anterior, se presenta la siguiente hipótesis:

$-\mathrm{H}_{5}$. La tendencia a la compra impulsiva tiene una influencia directa y positiva sobre la utilidad percibida en el comercio móvil.

\subsection{Necesidad de recursos materiales y orientación a la tarea}

La necesidad de recursos materiales ha sido definida por Mowen (2000) como el deseo de recolectar y poseer bienes materiales. Es un rasgo que deriva de la importancia que el propio individuo atribuye a las posesiones mundanas (Belk, 1984). Las necesidades de recursos materiales surgen de la combinación de posesión, falta de generosidad, envidia y preservación de la tangibilidad (Belk, 1984, 1985). 
Por otro lado, los individuos con tendencia a la compra impulsiva, como se ha visto anteriormente, suelen realizar compras de forma imprevista, repentina y no planeada como respuesta a estímulos subjetivos que los conducen a la satisfacción a través de la posesión inmediata (Rook, 1987). Dado que el materialismo está relacionado negativamente con el control de impulsos (Rose, 2007), cabe esperar que esta falta de control de los individuos con una alta necesidad de recursos materiales se vea reflejada en una tendencia a la compra impulsiva (Sun y Wu, 2011) y al endeudamiento (Yazdanparas y Alhenawi, 2017). En esta línea, la literatura existente sugiere una relación positiva entre el materialismo y la realización de compras impulsivas (Podoshen y Andrzejewski, 2012).

Por ello, proponemos la siguiente hipótesis:

$-\mathrm{H}_{6}$. La necesidad de recursos materiales influye directa y positivamente sobre la tendencia a la compra impulsiva.

Para los individuos con necesidades altas de recursos materiales, la felicidad y la satisfacción están directamente asociadas a sus posesiones materiales (Belk 1985; Yazdanparas y Alhenawi, 2017). Distintos autores han identificado relaciones positivas entre el materialismo y la emoción y la felicidad experimentadas tras la compra de un producto que para el individuo es importante (Millar y Thomas, 2009; Richins, 2013). Así, los consumidores con necesidades altas de recursos materiales perciben una mayor utilidad en aquellos mercados que les permiten realizar de manera eficiente la compra de los productos que son importantes para ellos (Kim et al., 2015). El comercio móvil, al dar acceso a toda la información sobre los productos que desean comprar, así como a la posibilidad de comparar precios y a realizar la compra sin tener que desplazarse (Aldás-Manzano et al., 2009), hará que los consumidores materialistas perciban este entorno como más útil.

De acuerdo con esto, se formula la siguiente hipótesis:

$-\mathrm{H}_{7}$. La necesidad de recursos materiales influye directa y positivamente sobre la utilidad percibida en el comercio móvil.

La orientación a la tarea viene definida como la disposición duradera a establecer objetivos, completar tareas y lograr altos niveles de rendimiento en su realización (Mowen, 2000).

Los individuos orientados a la tarea prefieren situaciones que impliquen afrontar retos desafiantes y que les permitan ampliar su nivel de conocimiento (Nicholls, 1984). Tienden a concentrar sus esfuerzos en el cumplimiento de las tareas y a ser independientes en sus comportamientos (Brown y Peterson, 1994). Los individuos centrados en completar las labores requeridas es probable que mantengan la atención en los detalles de rendimiento de la tarea, reconozcan la relación entre esfuerzo y rendimiento y perseveren en las acciones exigidas para un rendimiento provechoso (Spence y Helmreich, 1983). Así, la orientación a la tarea implica la valoración del esfuerzo (Seegers, van Putten y De Barbander, 2002) y se asocia a la consideración de que el esfuerzo lleva al éxito y a un mayor sentimiento de competencia a través del compromiso con la tarea (Nicholls, 1984, 1989). En este sentido, diversas investigaciones han encontrado una relación positiva entre la orientación a la tarea de los individuos y la competencia subjetiva del individuo o su nivel de esfuerzo (Brown y Peterson, 1994; Seegers et al., 2002). Cuando los individuos están involucrados en una tarea, su objetivo consiste en dominarla o aprender algo nuevo, por lo que es razonable considerar que se esforzarán más y experimentarán más éxito (Sarrazin, Roberts, Cury, Biddle y Famose, 2002). De este modo, cabe esperar que los individuos con una baja orientación a la tarea tenderán a evitar sistemas complicados que requieran esfuerzo cognitivo, mientras que los que tienen una alta orientación a la tarea tendrán expectativas y percepciones positivas sobre la facilidad de uso del nuevo sistema, ya que estarán más motivados a realizar un esfuerzo cognitivo.

De acuerdo con lo anterior, se propone la siguiente hipótesis:

$-\mathrm{H}_{8}$, La orientación a la tarea influye directa y positivamente sobre la percepción de facilidad de uso del comercio móvil. 
Por otro lado, los individuos con una elevada orientación a la tarea dan especial importancia a establecer y conseguir de forma provechosa sus objetivos (Mowen, 2000), reduciendo la importancia que otorgan a la satisfacción inmediata de impulsos. Son individuos que poseen la capacidad de centrar su atención en los objetivos que persiguen, ignorando los estímulos externos que los distraen de su consecución (Wills, Sandy y Yaeger, 2000).

Además, el materialismo se relaciona negativamente con el control de impulsos por parte de los individuos (Rose, 2007), que puede reflejarse en comportamientos de compra impulsivos. Puesto que una base sólida en los objetivos de los individuos es clave para mantener el autocontrol y no dejarse llevar por comportamientos impulsivos (Baumeister, 2002), se puede argumentar que la orientación a la tarea tendrá un efecto mitigador de la relación positiva entre la necesidad de recursos materiales y la tendencia a la compra impulsiva. Es probable que los individuos orientados a la tarea permanezcan centrados en esta y en los objetivos que persiguen (Tenenbaum et al., 2001), a pesar de los estímulos externos y de las situaciones a las que se enfrenten. En otras palabras, los individuos con una orientación a la tarea alta serán capaces de controlar su materialismo, reduciendo el efecto negativo que este tiene en el control de los estímulos y, por tanto, en la tendencia a la compra impulsiva.

Así, se presenta la siguiente hipótesis:

$-\mathrm{H}_{9}$. La orientación a la tarea modera el efecto positivo que la necesidad de recursos materiales tiene sobre la tendencia a la compra impulsiva, de forma que cuanto mayor sea la orientación a la tarea, menor será el efecto que la necesidad de recursos materiales tiene sobre la tendencia a la compra impulsiva.

La Figura 1 presenta el modelo propuesto en este estudio.

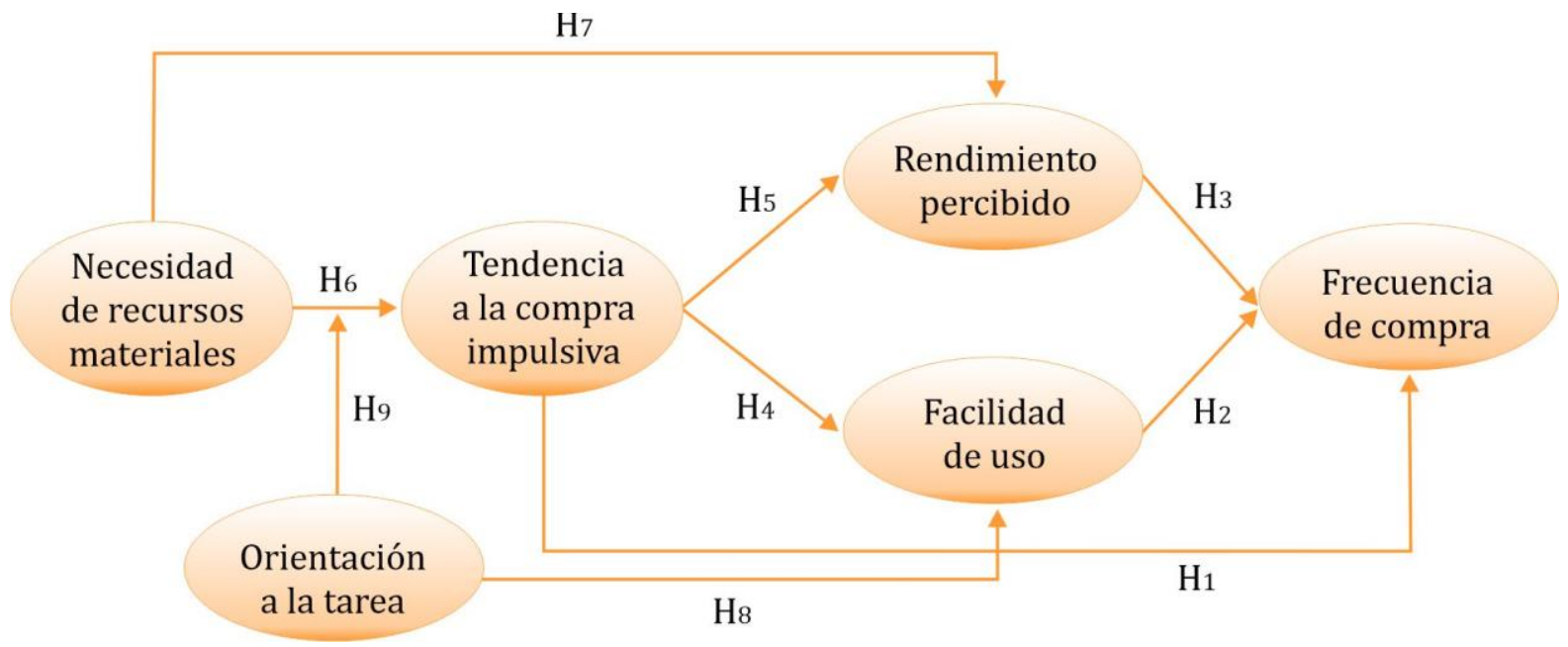

Figura 1. Modelo de relaciones propuesto. Fuente: elaboración propia.

\section{Metodología}

\subsection{Muestra}

La muestra empleada en la presente investigación para alcanzar los objetivos propuestos y contrastar las hipótesis expuestas está formada por individuos españoles que poseen al menos un dispositivo móvil con acceso a Internet. 
Para la obtención de los datos, se contactó de manera personal y online (mediante correo electrónico y las redes sociales Facebook e Instagram) con un total de 350 individuos. Una vez explicados los objetivos de la investigación y confirmado que los individuos poseían como mínimo un dispositivo móvil con conexión a Internet, se les envió el cuestionario a través del correo electrónico y de las redes sociales. En un plazo máximo de 30 días, los individuos debían devolver cubierto el cuestionario a través del mismo medio.

Los datos se han obtenido, por tanto, mediante encuestas personales con cuestionario estructurado realizado de forma autoadministrada. El número total de encuestas válidas ascendió a 208. Los datos se han obtenido a través de muestreo por conveniencia. La muestra está compuesta por un $62,5 \%$ de mujeres y un 37,5\% de hombres. El 55\% de los encuestados tienen entre 18 y 29 años, el $32 \%$ entre 30 y 39 años, el $9 \%$ entre 40 y 49 años y el $4 \%$ restante tienen 50 años o más.

\subsection{Escalas de medida}

Para medir los constructos empleados en esta investigación se han utilizado escalas de tipo Likert de cinco puntos, extraídas y adaptadas de la literatura previa específica en comercio móvil.

Las variables de orientación a la tarea y de necesidad de recursos materiales se han medido a través de cuatro ítems cada una, obtenidos de Sun y Wu (2011). La tendencia a la compra impulsiva se ha calculado a través de seis ítems extraídos de Rook y Fisher (1995). Las variables correspondientes al modelo TAM, facilidad de uso y utilidad percibida, han sido valoradas a través de cuatro ítems cada una, siguiendo a Venkatesh, Thong y Xu (2012). Por último, la frecuencia de compra se ha determinado a través de un único ítem, pidiendo a los encuestados lo siguiente: "Indique la frecuencia con la que ha realizado compras a través de su dispositivo móvil en los últimos seis meses", siendo $1=$ nunca y $5=a$ diario.

Las Tablas 1 y 2 recogen las escalas de medida empleadas y los estadísticos descriptivos.

\subsection{Propiedades psicográficas de las escalas}

El análisis factorial confirmatorio se ha realizado empleando EQS 6.1. Para evitar problemas de normalidad con los datos, se ha utilizado el método de máxima verosimilitud robusto para el análisis de los resultados.

La Tabla 1 recoge los resultados obtenidos para el modelo de medida. Los estimadores robustos de máxima verosimilitud muestran un adecuado ajuste general del modelo $\left(\mathrm{X}^{2}: 346,4410(\mathrm{gl}=231)\right.$ $p<, 001$; CFI: 0,934; RMSEA: 0,060). Los índices de ajuste se seleccionaron de acuerdo con las recomendaciones de la literatura psicométrica. Así, el CFI evalúa el aumento proporcional en el ajuste del modelo de medida sobre un modelo de base más restrictivo (Bentler, 1990; Bollen, 1989), y constituye uno de los índices de ajuste incremental más fiable y el más utilizado en la literatura de marketing (McDonald y Ho, 2002). El RMSEA hace referencia a la cantidad de varianza no explicada por el modelo por grado de libertad (Steiger y Lind, 1980), y supone una de las medidas más populares de bondad del ajuste en los modelos de ecuaciones estructurales (Kenny, Kaniskan y McCoach, 2015). Cuando se utilizan estos índices para evaluar el ajuste del modelo, valores próximos a 0,95 para el CFI e inferiores a 0,08 para el RMSEA indican un ajuste del modelo adecuado (Cudeck y Browne, 1993; Hair, Anderson, Tatham y Black, 2010; Hu y Bentler, 1999)

Por otra parte, consideramos que las escalas de medida empleadas son fiables, ya que el Alpha de Cronbach de todas las variables es superior a 0,7, la fiabilidad compuesta es superior a 0,6 y la varianza extraída media (AVE) es superior a 0,5 (Hair et al., 1999). Los indicadores utilizados son válidos, ya que se confirma la validez convergente -el valor de las cargas estandarizadas es superior a 0,5 y son estadísticamente significativos- y la validez discriminante -las correlaciones entre variables dan lugar a intervalos de confianza que no incluyen la unidad (Anderson y Gerbing, 1988), y su valor al cuadrado es inferior al AVE de los factores implicados (Fornell y Larcker, 1981)-, como se muestra en la Tabla 2. 
Tabla 1. Propiedades psicométricas de las variables

Indicador

Carga

estand. $(*)$

TENDENCIA A LA COMPRA IMPULSIVA: $(\alpha=0,860 ;$ IFC $=0,862 ;$ AVE $=0,514)$

- A menudo compro cosas de forma espontánea.

- "Solo hazlo" describe la forma en que compro cosas.

$0,720^{*}$

- "Lo veo, lo compro" me describe.

- "Compra ahora, piénsalo después" me describe.

$0,817^{*}$

- Compro cosas de acuerdo a cómo me sienta en ese momento.

$0,750 *$

- A veces soy un poco temerario con lo que compro.

$0,654^{*}$

$0,588^{*}$

UTILIDAD PERCIBIDA: $(\alpha=0,885 ;$ IFC $=0,888 ;$ AVE $=0,667)$

- Creo que la compra/pago a través de un dispositivo móvil es útil en la vida diaria.

$0,720^{*}$

- El uso de la compra/pago a través de un dispositivo móvil aumenta las posibilidades de lograr

$0,858^{*}$ cosas que son importantes para mí.

- El uso de la compra/pago a través de un dispositivo móvil facilita lograr cosas con mayor rapi-

0,733* dez.

- El uso de la compra/pago a través de un dispositivo móvil mejora mi efectividad.

$0,936^{*}$

FACILIDAD DE USO: $(\alpha=0,906 ;$ IFC $=0,907 ;$ AVE $=0,710)$

- Aprender a usar el método de compra/pago a través de un dispositivo móvil me parece fácil.

- La compra/pago a través de un dispositivo móvil es comprensible y clara.

- La compra/pago a través de un dispositivo móvil es fácil de usar.

$0,867 *$

- Me resultaría fácil alcanzar destreza en la realización de compras/pagos a través de un disposi-

$0,811^{*}$ tivo móvil.

NECESIDAD DE RECURSOS MATERIALES: $(\alpha=0,892$; IFC $=0,896$; AVE $=0,700)$

- Me gusta tener cosas buenas más que a la mayoría de la gente.

- Disfruto comprando cosas caras.

- Adquirir cosas valiosas es importante para mí.

$0,854^{*}$

- Disfruto teniendo cosas lujosas.

$0,843^{*}$

ORIENTACIÓN A LA TAREA: $(\alpha=0,789 ;$ IFC $=0,806$; AVE $=0,521)$.

- Estoy orientado a metas a largo plazo.

- Al realizar una tarea, establezco un plazo para la finalización.

- Establezco metas a largo plazo para el futuro.

$0,914^{*}$

- Afronto las tareas de manera seria.

$0,500^{*}$

Nota: Satorra-Bentler X²: 346,4410 (gl = 231) $p<, 001$; CFI: 0,934; RMSEA: 0,060; * $p<0,01$. Fuente: elaboración propia.

Tabla 2. Validez discriminante

\begin{tabular}{lccccc}
\hline & 1 & 2 & 3 & 4 & 5 \\
\hline 1. Tendencia a la compra impulsiva & 0,514 & {$[0,295-0,581]$} & {$[0,092-0,386]$} & {$[0,413-0,687]$} & {$[-0,102-0,216]$} \\
2. Utilidad percibida & 0,192 & 0,667 & {$[0,378-0,624]$} & {$[0,207-0,473]$} & {$[-0,018-0,300]$} \\
3. Facilidad de uso & 0,057 & 0,251 & 0,710 & {$[-0,076-0,234]$} & {$[0,136-0,414]$} \\
4. Necesidad de recursos materiales & 0,303 & 0,116 & 0,006 & 0,700 & {$[-0,109-0,193]$} \\
5. Orientación a la tarea & 0,003 & 0,020 & 0,076 &, 002 & 0,521 \\
Media & 2,122 & 3,566 & 3,948 & 2,088 & 3,426 \\
Desviación típica &, 897 & 1,055 &, 883 &, 927 &, 827 \\
\hline
\end{tabular}

Nota: los elementos de la diagonal (en color naranja) muestran la AVE. Se presentan las correlaciones al cuadrado. Fuente: elaboración propia. 


\section{Resultados}

El conjunto de hipótesis que constituyen el modelo propuesto en esta investigación implica un proceso de mediación moderado (Edwards y Lambert, 2007; Bauer, Preacher y Gil, 2006). Para realizar su evaluación, se han seguido los pasos descritos por Preacher, Rucker y Hayes (2007). Con el objetivo de simplificar el modelo propuesto, los constructos han sido sustituidos por la media de los indicadores. Dado que las escalas de medida empleadas para algunas variables del modelo no incluyen el valor cero, las variables involucradas en los términos de interacción han sido centradas a la media con el fin de evitar problemas en la interpretación de sus coeficientes. Finalmente, y de cara a evitar los efectos de confusión de otras variables que puedan estar relacionadas con el resultado analizado, controlamos por edad y por sexo del individuo. La Tabla 3 recoge los resultados del modelo de ecuaciones estructurales utilizado para realizar los análisis de las hipótesis propuestas.

Tabla 3. Resultados del análisis

\begin{tabular}{lc}
\hline Variables & Coeficientes \\
\hline FRECUENCIA DE COMPRA $\left(R^{2}=, 389\right)$ &, $405^{* *}$ \\
Utilidad percibida &, $287^{* *}$ \\
Facilidad de uso &, $245^{* *}$ \\
Tendencia a la compra impulsiva &,- 075 \\
Edad &, 167 \\
Sexo &, 064 \\
Orientación a la tarea $(O)$ &,- 030 \\
Necesidad de recursos materiales $(N)$ &, 003 \\
$O \times N$ & \\
UTILIDAD PERCIBIDA $\left(R^{2}=, 215\right)$ &, $339^{* *}$ \\
Tendencia a la compra impulsiva &,$- 236^{* *}$ \\
Edad &, 026 \\
Sexo &, 109 \\
Orientación a la tarea $(O)$ &, $174^{*}$ \\
Necesidad de recursos materiales $(N)$ &,- 034 \\
$O \times N$ & \\
FACILIDAD DE USO $\left(R^{2}=, 144\right)$ & \\
Tendencia a la compra impulsiva &, $165^{*}$ \\
Edad &, $222^{* *}$ \\
Sexo &, 112 \\
Orientación a la tarea $(O)$ &, 101 \\
Necesidad de recursos materiales $(N)$ &, $272^{* *}$ \\
$O \times N$ &,- 031 \\
TENDENCIA A LA COMPRA IMPULSIVA $\left(R^{2}=, 311\right)$ &,- 110 \\
Edad & \\
Sexo &,$- 181^{* *}$ \\
Orientación a la tarea $(O)$ &,- 205 \\
Necesidad de recursos materiales $(N)$ &,- 032 \\
$O \times N$ &, 6336 \\
\hline & \\
\hline & \\
\hline & \\
&
\end{tabular}

Nota: ${ }^{* *} p<, 01 ;{ }^{*} p<, 05$. Fuente: elaboración propia. 
Para las relaciones propuestas en las hipótesis 1, 2 y 3 entre la tendencia a la compra impulsiva y la frecuencia de compra en el comercio móvil y entre la utilidad percibida y la facilidad de uso y la frecuencia de compra en el comercio móvil, todas las hipótesis se cumplen. Tanto la tendencia a la compra impulsiva como la utilidad percibida y la facilidad de uso tienen un efecto positivo sobre la frecuencia de compra en el comercio móvil.

En lo relativo a las relaciones entre la tendencia a la compra impulsiva y la facilidad de uso y la utilidad percibida propuestas en las hipótesis 4 y 5 , podemos confirmar su cumplimiento. La tendencia a la compra impulsiva tiene un efecto positivo sobre ambas variables: utilidad percibida y facilidad de uso.

Los resultados obtenidos del modelo de ecuaciones estructurales en cuanto a las hipótesis 6 y 7 , propuestas para la influencia de la necesidad de recursos materiales sobre la tendencia a la compra impulsiva y la utilidad percibida, también confirman su cumplimiento. La necesidad de recursos materiales tiene un efecto positivo sobre la tendencia a la compra impulsiva y sobre la utilidad percibida.

Por otra parte, la relación propuesta en la hipótesis 8 entre la orientación a la tarea y la facilidad de uso también se soporta. Por tanto, la orientación a la tarea tiene un efecto positivo sobre la facilidad de uso del comercio móvil.

Por lo que respecta a la hipótesis 9, referida al efecto moderador de la orientación a la tarea sobre la relación entre la necesidad de recursos materiales y la tendencia a la compra impulsiva, los resultados obtenidos reflejan una relación significativa, pero de signo contrario al propuesto. En este sentido, se ha obtenido un efecto moderador y positivo de la orientación a la tarea en la relación entre la necesidad de recursos materiales y la tendencia a la compra impulsiva. En la Figura 2 se presenta la interacción, empleando el proceso utilizado por Dawson (2014). Se observa que altos niveles de orientación a la tarea incrementan el efecto positivo que la necesidad de recursos materiales tiene sobre la tendencia a la compra impulsiva.

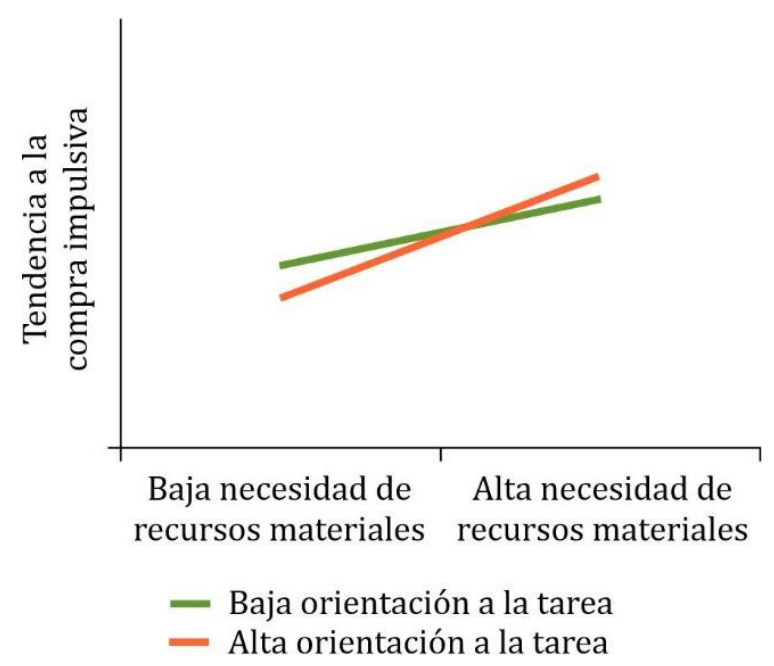

Figura 2. Interacción orientación a la tarea-necesidad de recursos materiales. Fuente: elaboración propia.

Para evaluar la mediación moderada es necesario comprobar si la fuerza de la mediación por la vía de la tendencia a la compra impulsiva, de la facilidad de uso y de la utilidad percibida varía para diferentes niveles de la orientación a la tarea, análisis que constituye la esencia de la mediación moderada. Para ello se emplearon intervalos de confianza bootstrapped del 95\% (derivados de 5.000 replicaciones), de acuerdo con Preacher et al. (2007). En este sentido, se ha calculado la significación de las relaciones indirectas entre la necesidad de recursos materiales y la frecuencia de compra en el comercio 
móvil por la vía de la tendencia a la compra impulsiva, de la tendencia a la compra impulsiva y de la facilidad de uso, y también por la vía de la tendencia a la compra impulsiva y de la utilidad percibida. En todos los casos, se ha estimado para consumidores con una orientación a la tarea baja, moderada y alta. Adicionalmente, se han evaluado las significaciones de las relaciones indirectas entre la orientación a la tarea y la frecuencia de compra por la vía de la facilidad de uso, y entre las necesidades de recursos materiales y la frecuencia de compra en comercio móvil mediante la utilidad percibida.

Los resultados (Tabla 4) reflejan que la relación indirecta condicionada "necesidad de recursos materiales $\rightarrow$ tendencia a la compra impulsiva $\rightarrow$ frecuencia de compra en comercio móvil" es positiva y significativa para todos los niveles de orientación a la tarea, siendo más fuerte para el nivel alto. Asimismo, las relaciones indirectas condicionadas "necesidad de recursos materiales $\rightarrow$ tendencia a la compra impulsiva $\rightarrow$ facilidad de uso percibida $\rightarrow$ frecuencia de compra en comercio móvil" y "necesidad de recursos materiales $\rightarrow$ tendencia a la compra impulsiva $\rightarrow$ utilidad percibida $\rightarrow$ frecuencia de compra en comercio móvil" también son positivas y significativas en los niveles moderado y alto, siendo estas relaciones más fuertes cuanto más alto es el nivel. Por último, las relaciones indirectas "necesidad de recursos materiales $\rightarrow$ utilidad percibida $\rightarrow$ frecuencia de compra en comercio móvil" y "orientación a la tarea $\rightarrow$ facilidad de uso percibida $\rightarrow$ frecuencia de compra en comercio móvil" también son positivas e significativas.

Tabla 4. Test de los efectos indirectos de necesidad de recursos materiales y orientación a la tarea sobre la frecuencia de compra en comercio móvil

\begin{tabular}{|c|c|c|c|c|c|}
\hline Efectos indirectos & $\begin{array}{l}\text { Orientación } \\
\text { a la tarea }\end{array}$ & Efecto & $\begin{array}{l}\text { Boot } \\
\text { SE }\end{array}$ & $\begin{array}{l}\text { Bias-corrected } \\
\text { LLCI }\end{array}$ & $\begin{array}{l}\text { Bias-corrected } \\
\text { ULCI }\end{array}$ \\
\hline $\begin{array}{l}\text { Necesidad de recursos materiales } \rightarrow \text { Tenden- } \\
\text { cia la compra impulsiva } \rightarrow \text { Frecuencia de } \\
\text { compra. }\end{array}$ & $\begin{array}{c}\text { Bajo } \\
\text { Moderado } \\
\text { Alto }\end{array}$ & $\begin{array}{l}, 083^{* *} \\
, 117^{* *} \\
, 151^{* *}\end{array}$ & $\begin{array}{l}, 041 \\
, 053 \\
, 072\end{array}$ & $\begin{array}{l}, 022 \\
, 025 \\
, 030\end{array}$ & $\begin{array}{l}190 \\
, 234 \\
320\end{array}$ \\
\hline $\begin{array}{l}\text { Necesidad de recursos materiales } \rightarrow \text { Tenden- } \\
\text { cia a la compra impulsiva } \rightarrow \text { Facilidad de uso } \\
\rightarrow \text { Frecuencia de compra. }\end{array}$ & $\begin{array}{l}\text { Bajo } \\
\text { Moderado } \\
\text { Alto }\end{array}$ & $\begin{array}{l}, 022 \\
, 030^{*} \\
, 039^{*}\end{array}$ & $\begin{array}{l}, 013 \\
, 017 \\
, 022\end{array}$ & $\begin{array}{l}, 005 \\
, 007 \\
, 009\end{array}$ & $\begin{array}{l}, 064 \\
, 075 \\
, 010\end{array}$ \\
\hline $\begin{array}{l}\text { Necesidad de recursos materiales } \rightarrow \text { Tenden- } \\
\text { cia a la compra impulsiva } \rightarrow \text { Utilidad percibida } \\
\rightarrow \text { Frecuencia de compra. }\end{array}$ & $\begin{array}{c}\text { Bajo } \\
\text { Moderado } \\
\text { Alto }\end{array}$ & $\begin{array}{l}, 047^{*} \\
, 065^{* *} \\
, 084^{* *}\end{array}$ & $\begin{array}{l}, 025 \\
, 029 \\
, 038\end{array}$ & $\begin{array}{l}, 015 \\
, 023 \\
, 029\end{array}$ & $\begin{array}{l}\text {,114 } \\
138 \\
, 183\end{array}$ \\
\hline $\begin{array}{l}\text { Necesidad de recursos materiales } \rightarrow \text { Utilidad } \\
\text { percibida } \rightarrow \text { Frecuencia de compra. }\end{array}$ & - &, $070^{*}$ & ,037 & ,009 & ,158 \\
\hline $\begin{array}{l}\text { Orientación a la tarea } \rightarrow \text { Facilidad de uso } \rightarrow \\
\text { Frecuencia de compra. }\end{array}$ & - &, $078^{* * *}$ & ,029 & ,033 & ,150 \\
\hline
\end{tabular}

Notas: ${ }^{* *} p<, 01 ;{ }^{* *} p<, 05 ;{ }^{* *} p<, 10$. Bootstrapped CIs fueron derivados de 5.000 replicaciones. Alto $=1$ SD por encima de la media; Moderado = media; Bajo = 1SD por debajo de la media. Fuente: elaboración propia.

\section{Conclusiones}

Múltiples autores han señalado la importancia de la personalidad de los individuos sobre los comportamientos de compra, tanto dentro como fuera del comercio móvil (Bosnjak, Galesic y Tuten, 2007; Miao, Jalees, Qabool y Zaman, 2019; Putrawan, 2020; Shahjehan, Zeb y Saifullah, 2012). En este senti- 
do, este trabajo trata de alcanzar un conocimiento más completo de la influencia de los rasgos de personalidad sobre el comportamiento de compra de los individuos en el comercio móvil.

La investigación realizada pone de manifiesto la efectividad del modelo propuesto y su adecuada contribución a la limitada investigación existente en el ámbito del comercio móvil. A través de la fusión de los rasgos de personalidad y el modelo TAM, los resultados obtenidos ponen de manifiesto la gran importancia de los rasgos de la personalidad de los individuos, en concreto las necesidades de recursos materiales, la orientación a la tarea o la tendencia a la compra impulsiva, sobre la frecuencia de compra en el entorno del comercio móvil.

Con el desarrollo tecnológico y la consecuente proliferación de entornos de compra online y móvil, las compras impulsivas se han convertido en un fenómeno emergente (Chen, Su y Widjaja, 2016; Liu y Xiao, 2018). Los resultados de este estudio, de conformidad con las argumentaciones existentes en los análisis sobre compra impulsiva (p.e., Lim, Lee y Kim, 2017; Zheng et al., 2019), recogen un importante efecto positivo de la tendencia a la compra impulsiva sobre la frecuencia de compra de los individuos en el comercio móvil. Las características propias del medio (Lee, Park y Jun,, 2014), así como los múltiples y efectivos estímulos disponibles (Stefańska y Śmigielska, 2020) en el comercio móvil, generarán en los compradores impulsivos una mayor frecuencia de compra. En este campo, la presente investigación contribuye a una mejor comprensión de la tendencia a la compra impulsiva.

Adicionalmente, el estudio realizado confirma la influencia positiva de la tendencia a las compras impulsivas sobre los constructos principales del TAM: facilidad de uso y utilidad percibida. Estos resultados suponen una novedad respecto de las investigaciones previas, que centraban su atención en el comportamiento de compra impulsiva (p.e., Lee, 2018) en lugar de en el rasgo de personalidad que impulsa la tendencia a dicha compra. La accesibilidad total e inmediata que permite el comercio móvil a la hora de efectuar compras en cualquier lugar y momento (Kim et al., 2015), con el ahorro en tiempo y esfuerzo que esto implica, sumado a las herramientas disponibles que permiten la satisfacción de los impulsos de los consumidores de manera sencilla y rápida (Wong et al., 2012) son aspectos que generarán una gran percepción de utilidad y facilidad de uso del comercio móvil en aquellas personas con mayor tendencia a la compra impulsiva.

Por otra parte, resulta interesante destacar la influencia positiva que los otros rasgos de personalidad analizados tienen sobre las percepciones de los individuos. En este punto, los resultados alcanzados reflejan un efecto positivo de la necesidade de recursos materiales sobre la utilidad percibida, y de la orientación a la tarea sobre la facilidad de uso del comercio móvil. El efecto de la necesidad de recursos materiales sobre la utilidad percibida de los individuos está de acuerdo con las argumentaciones propuestas en análisis previos (p.e., Ziano y Villanova, 2019) acerca del efecto de aspectos del materialismo sobre la percepción de utilidad de las personas. La disposición de información actualizada y relevante sobre los productos y servicios, junto con la posibilidad de comparar precios así como de realizar las compras sin necesidad de desplazarse (Aldás-Manzano et al., 2009), generarán una elevada percepción de utilidad del comercio móvil por parte de los individuos con altas necesidades de recursos materiales, al permitirles adquirir posesiones materiales de manera más eficiente (Kim et al., 2015).

En relación con el efecto positivo de la orientación a la tarea sobre la facilidad de uso, los resultados obtenidos están en línea con los alcanzados por trabajos previos (Brown y Peterson, 1994; Seegers et al., 2002). Las múltiples herramientas y sistemas de ayuda disponibles en el comercio móvil (Groß, 2015; Kim et al., 2015), sumadas a la perseverancia y a la motivación de los individuos con alta orientación a la tarea a realizar esfuerzos cognitivos, generarán una mayor percepción de facilidad de uso del comercio móvil por parte de estas personas. De acuerdo con esto, la investigación realizada contribuye a una comprensión más completa del funcionamiento del modelo TAM, a través del estudio de antecedentes de personalidad en él.

Además, de conformidad con las argumentaciones existentes en la literatura, los resultados conseguidos reflejan una relación positiva entre las necesidades materiales y la tendencia a las compras impulsivas (Barakat, 2019; Parsad, Prashar y Tata, 2019; Podoshen y Andrzejewski, 2012; Šeinauskienė, Maščinskienè, Petrike y Rutelionė, 2016; Sun y Wu, 2011). La importancia de almacenar y poseer bie- 
nes, junto con la falta de control de impulsos de los individuos con altas necesidades de recursos materiales (Rose, 2007), contribuirán a una mayor tendencia a la compra impulsiva. Con el objetivo de completar la comprensión de dicha relación, se ha analizado la influencia moderadora de la orientación a la tarea sobre tal impulso de compra. Los resultados, en contradicción con lo esperado, reflejan un incremento del efecto positivo de la necesidad de los recursos materiales sobre la tendencia a la compra impulsiva cuando el individuo cuenta con una elevada orientación a la tarea. En otras palabras, los resultados muestran que la conjunción en los individuos de la necesidad de poseer bienes materiales con la tendencia a establecer objetivos y a preocuparse por completarlos generará una mayor tendencia a la compra impulsiva. Este resultado puede venir motivado por el momento de la experiencia de compra en que se encuentra el individuo. Las compras impulsivas se caracterizan por la ausencia de objetivos destacados, al menos al comienzo de la experiencia (Peck y Childers, 2006), de lo que cabría esperar que la conjunción en los individuos de la necesidad de recursos materiales con la orientación a la tarea redujese el efecto positivo de la necesidad de recursos materiales sobre la tendencia a la compra impulsiva. No obstante, cabe la posibilidad de que las personas se encuentren, al comienzo, en una fase de la experiencia de compra diferente en la cual existan objetivos salientes. En este caso, una mayor orientación a la tarea por parte de los individuos potenciaría el efecto positivo de las necesidades de recursos materiales sobre la tendencia a la compra impulsiva, no solo por la satisfacción que generaría la obtención de posesiones materiales, sino también por la satisfacción que produciría la consecución de los objetivos propuestos.

Asimismo, y en línea con investigaciones previas que reflejan una relación positiva entre el materialismo y la compra (p.e., Kamal, Chu y Pedram, 2013; Sun, Wang, Cheng, Li y Chen, 2017), los resultados alcanzados recogen una relación positiva e indirecta entre la necesidad de recursos materiales y la frecuencia de compra en el comercio móvil, a través de la tendencia a la compra impulsiva, a la facilidad de uso y a la utilidad percibida, dependiendo dichas relaciones del nivel de orientación a la tarea que presentan los individuos. En concreto, la influencia indirecta de la necesidad de recursos materiales sobre la frecuencia de compra será mayor cuanto mayor sea la orientación a la tarea. Adicionalmente, los resultados reflejan un efecto indirecto y positivo de la orientación a la tarea sobre la frecuencia de compra (a través de la facilidad de uso) y un efecto indirecto y positivo de la necesidad de recursos materiales sobre la frecuencia de compra en el comercio móvil (a través de la utilidad percibida). Estos resultados contribuyen a la literatura del comercio móvil a través de la fusión de los rasgos de personalidad con el modelo TAM, ya que todavía son escasas las investigaciones que analizan el efecto de la personalidad sobre el comportamiento de compra en el ámbito del comercio móvil (Agyei et al., 2020; Lissitsa y Kol, 2019).

\section{Implicaciones empresariales}

Las múltiples posibilidades de negocio y acción que permite el comercio móvil, tanto para empresas como para consumidores, han centrado la atención de muchos investigadores (Huang y Zhou, 2018; Kim et al., 2017) con el objetivo de ofrecer a las empresas pautas de seguimiento adecuadas para mejorar su situación y alcanzar ventajas competitivas que les permitan despuntar frente a otras empresas. Los resultados de esta investigación ponen de relieve una serie de implicaciones para las empresas, cuyo objetivo central es el incremento de la frecuencia de compra de sus consumidores.

Los efectos que las necesidades de recursos materiales, la orientación a la tarea y la tendencia a la compra impulsiva tienen sobre la frecuencia de compra de los individuos en el comercio móvil ponen de manifiesto la necesidad de que las empresas tengan muy presente la personalidad de los consumidores a los que se orientan y a los que quieren atraer, permitiendo así adaptar sus plataformas, y los servicios y productos que ofrecen, con el objetivo de lograr un incremento en las compras. Los efectos positivos que la necesidad de recursos materiales tiene sobre la frecuencia de compra de los individuos en el comercio móvil muestran la exigencia de que las empresas faciliten a estos consumidores el acceso a través del comercio móvil a la totalidad de sus productos. Las empresas pueden emplear 
pop-ups o ventanas emergentes (Kim, 2018), con productos adaptados en función de las búsquedas y compras realizadas, a través de las cuales se potenciaría la falta de control de impulsos de los individuos con altas necesidades de recursos materiales (Rose, 2007).

Además, las empresas deben tratar de mejorar la eficiencia del proceso de compra a través del comercio móvil, incorporando más información relevante sobre los productos -por ejemplo, comentarios de otros consumidores que hayan adquirido previamente un producto y fotografías reales de este (Li, Huang, Tan y Wei, 2013)- que proporcionen a los individuos información de utilidad para la compra. Igualmente, deben utilizar herramientas que permitan la comparación de precios con los de la competencia, o incorporar la opción de "compra en un click" (Chen y Lan, 2018), que recuerda los datos del consumidor y permite realizar la compra ahorrando tiempo y esfuerzo. Analizando el uso de la opción de compra en un click, las empresas podrían identificar a los consumidores con altas necesidades de recursos materiales y tratar de potenciar este rasgo mediante el uso de pop-ups con productos personalizados o a través de sugerencias u ofertas especiales de productos que se adapten a sus gustos.

Los efectos de la orientación a la tarea sobre la frecuencia de compra en el comercio móvil reflejan la necesidad de que las empresas contribuyan a la consecución por parte de los consumidores de sus objetivos de manera eficiente. En este campo, las empresas pueden poner a disposición de los consumidores las listas de deseos con herramientas específicas, aún ausentes en muchas aplicaciones y plataformas de comercio móvil. A través de las listas de deseos, los individuos pueden establecer sus objetivos de adquisición y planificar sus compras para completarlas con un mayor nivel de rendimiento (Ambre, Gaikwad, Pawar y Patil, 2019). Asimismo, la incorporación de herramientas específicas en las listas de deseos, que muestren por ejemplo la evolución experimentada por los precios en los últimos 30 días de los diferentes productos añadidos, o las promociones existentes para el conjunto de productos de la lista, así como un apartado de productos sugeridos basado en los productos presentes en la lista de deseos, podrían contribuir a una consecución más eficiente de los objetivos por parte de los individuos, mejorando así su orientación a la tarea y repercutiendo positivamente sobre la frecuencia de compra. A través del análisis de estas listas de deseos, y su posterior evolución a la fase de compra, las empresas podrían identificar a los consumidores con una elevada orientación a la tarea y tratar de potenciar dicho rasgo.

Por otra parte, los resultados destacan la importancia que la tendencia a las compras impulsivas tiene en la frecuencia de compra en el comercio móvil. Las empresas deben tratar de aprovechar al máximo los medios disponibles en los dispositivos móviles y en las aplicaciones para hacer llegar con frecuencia a los consumidores con esta tendencia diversos estímulos incentivadores que incrementen el atractivo de los bienes y servicios que ofertan (Parsad, Prashar, Vijay y Kumar, 2018). Al mismo tiempo, deberán usar estos medios para generar en los consumidores con esta tendencia un deseo de compra inmediata que no encuentre obstáculos a la hora de verse satisfecho.

Los resultados muestran que las percepciones de los individuos acerca de la accesibilidad temporal y geográfica, de la amplitud de opciones disponibles o de la variedad y amplitud de información y facilidad de uso (tanto en el acceso como en la navegabilidad) ofrecidas por el comercio móvil incrementarán la frecuencia de compra en este mercado. Por tanto, las empresas que desean incrementar sus ingresos por ventas a través de una mayor frecuencia de compra deberán tratar de poner a disposición de sus usuarios plataformas que les aporten un alto rendimiento, sin descuidar la sencillez a la hora de acceder a ellas y manejarlas.

\section{Limitaciones y líneas de investigación futuras}

Finalmente, debemos mencionar que nuestro estudio presenta limitaciones que deben tenerse en cuenta a la hora de tratar los resultados obtenidos.

En primer lugar, la muestra empleada en la investigación refleja una sobrerrepresentación del segmento de mujeres y del segmento de población más joven, de entre 18 y 29 años, respecto de la pobla- 
ción de estudio, de acuerdo con el perfil del comprador online presentado por el Observatorio Nacional de las Telecomunicaciones y de la Sociedad de la Información (ONTSI) en el año 2019. Estudios futuros podrían analizar las relaciones propuestas empleando para ello una muestra más representativa del perfil del consumidor online.

En segundo lugar, la muestra empleada para el estudio está constituida únicamente por individuos españoles. Es posible que los rasgos de personalidad, comportamientos y percepciones analizadas puedan diferir en función del país. Futuras investigaciones deberían examinar estas diferencias entre consumidores de diferentes culturas.

En tercer lugar, se han empleado encuestas para la obtención de los datos con los que se ha trabajado. En el futuro podrían emplear métodos experimentales y cualitativos para la obtención de los datos, contribuyendo así a una comprensión más completa de las relaciones analizadas.

En cuarto lugar, esta investigación emplea datos de carácter transversal, lo cual supone un problema a la hora de realizar inferencias causales. Otros eventuales análisis podrían replicar el estudio utilizando en su lugar datos longitudinales que proporcionen una información más completa.

\section{Bibliografía}

Adams, D. A., Nelson, R. R., y Todd, P. A. (1992). Perceived usefulness, ease of use, and usage of information technology: A replication. MIS Quarterly, 16(2), 227-247. DOI: https://doi.org/10.2307/249577

Agyei, J., Sun, S., Abrokwah, E., Penney, E. K., y Ofori-Boafo, R. (2020). Mobile banking adoption: Examining the role of personality traits. SAGE Open, 10(2), 1-15. DOI: https://doi.org/10.1177/2158244020932918

Alalwan, A. A., Algharabat, R. S., Baabdullah, A. M., Rana, N. P., Qasem, Z., y Dwivedi, Y. K. (2020). Examining the impact of mobile interactivity on customer engagement in the context of mobile shopping. Journal of Enterprise Information Management, 33(3), 627-653. DOI: https://doi.org/10.1108/JEIM-07-2019-0194

Aldás-Manzano, J., Ruiz-Mafé, C., y Sanz-Blas, S. (2009). Exploring individual personality factors as drivers of M-shopping acceptance. Industrial Management \& Data Systems, 109(6), 739-757. DOI: https://doi.org/10.1108/02635570910968018

Al-Samarraie, H., Eldenfria, A., y Dawoud, H. (2017). The impact of personality traits on users' information-seeking behavior. Information Processing \& Management, 53(1), 237-247. DOI: https://doi.org/10.1016/j.ipm.2016.08.004

Ambre, A., Gaikwad, P., Pawar, K., y Patil, V. (2019). Web and android application for comparison of e-commerce products. International Journal of Advanced Engineering, Management and Science, 5(4), 266-268. DOI: https://doi.org/10.22161/ijaems.5.4.5

Anderson, J. C., y Gerbing, D. W. (1988). Structural equation modeling in practice: A review and recommended two-step approach. Psychological Bulletin, 103(3), 411. DOI: https://doi.org/10.1037/0033-2909.103.3.411

Ashraf, A. R., Thongpapanl, N., Menguc, B., y Northey, G. (2017). The role of m-commerce readiness in emerging and developed markets. Journal of International Marketing, 25(2), 25-51. DOI: https://doi.org/10.1509/iim.16.0033

Aydın, G. (2019). Do personality traits and shopping motivations affect social commerce adoption intentions? Evidence from an emerging market. Journal of Internet Commerce, 18(4), 428-467. DOI: https://doi.org/10.1080/15332861.2019.1668659

Barakat, M. A. (2019). A proposed model for factors affecting consumers' impulsive buying tendency in shopping malls. Journal of Marketing Management, 7(1), 120-134.

Recuperado de: http://imm-net.com/journals/imm/Vol 7 No 1 June 2019/10.pdf

Barnett, T., Pearson, A. W., Pearson, R., y Kellermanns, F. W. (2015). Five-factor model personality traits as predictors of perceived and actual usage of technology. European Journal of Information Systems, 24(4), 374-390. DOI: https://doi.org/10.1057/ejis.2014.10

Bauer, D. J., Preacher, K. J., y Gil, K. M. (2006). Conceptualizing and testing random indirect effects and moderated mediation in multilevel models: New procedures and recommendations. Psychological Methods, 11(2), 142-163. DOI: https://doi.org/10.1037/1082-989X.11.2.142

Baumeister, R. F. (2002). Yielding to temptation: Self-control failure, impulsive purchasing, and consumer behavior. Journal of Consumer Research, 28(4), 670-676. DOI: https://doi.org/10.1086/338209

Baumeister, R. F., Heatherton, T. F., y Tice, D. M. (1994). Losing control: How and why people fail at selfregulation. San Diego, CA: Academic Press. 
Belk, R. W. (1984). Three scales to measure constructs related to materialism: Reliability, validity, and relationships to measures of happiness. En T. C. Kinnear (Ed.), Advances in Consumer Research, 11 (pp. 291-297). Provo, UT: Association for Consumer Research. Recuperado de: https://www.acrwebsite.org/volumes/6260/volumes/v11/NA-11

Belk, R. W. (1985). Materialism: Trait aspects of living in the material world. Journal of Consumer Research, 12(3), 265-280. DOI: https://doi.org/10.1086/208515

Bentler, P. M. (1990). Comparative fit indices in structural models, Psychological Bulletin, 107(2), 238-246. DOI: https://doi.org/10.1037/0033-2909.107.2.238

Bollen, K. A. (1989). A new incremental fit index for general structural equation models. Sociological Methods \& Research, 17(3), 303-316. D0I: https://doi.org/10.1177/0049124189017003004

Bosnjak, M., Galesic, M., y Tuten, T. (2007). Personality determinants of online shopping: Explaining online purchase intentions using a hierarchical approach. Journal of Business Research, 60(6), 597-605. DOI: https://doi.org/10.1016/i.jbusres.2006.06.008

Brown, S. P., y Peterson, R. A. (1994). The effect of effort on sales performance and job satisfaction. Journal of Marketing, 58(2), 70-80. DOI: https://doi.org/10.1177/002224299405800206

Bruner, G. C., y Kumar, A. (2005). Explaining consumer acceptance of handheld Internet devices. Journal of Business Research, 58(5), 553-558. D0I: https://doi.org/10.1016/j.jbusres.2003.08.002

Burton-Jones, A., y Hubona, G. S. (2006). The mediation of external variables in the technology acceptance model. Information \& Management, 43(6), 706-717. DOI: https://doi.org/10.1016/j.im.2006.03.007

Chang, A., Hsieh, S. H., y Lin, F. (2013). Personality traits that lead members of online brand communities to participate in information sending and receiving. International Journal of Electronic Commerce, 17(3), 37-62. DOI: https://doi.org/10.2753/JEC1086-4415170302

Chen, J. V., Su, B.-C., y Widjaja, A. E. (2016). Facebook C2C social commerce: A study of online impulse buying. Decision Support Systems, 83, 57-69. DOI: https://doi.org/10.1016/i.dss.2015.12.008

Chen, Y. F., y Lan, Y. C. (2018). An empirical study of the factors affecting mobile shopping in Taiwan. In Mobile Commerce: Concepts, methodologies, tools, and applications (pp. 1329-1340). Hershey, PA: IGI Global. DOI: https://doi.org/10.4018/978-1-5225-2599-8.ch063

Chen, Y. M., Hsu, T. H., y Lu, Y. J. (2018). Impact of flow on mobile shopping intention. Journal of Retailing and Consumer Services, 41, 281-287. DOI: https://doi.org/10.1016/j.jretconser.2017.04.004

Chong, A. Y. L. (2013). Mobile commerce usage activities: The roles of demographic and motivation variables. Technological Forecasting and Social Change, 80(7), 1350-1359.

DOI: https://doi.org/10.1016/i.techfore.2012.12.011

Chopdar, P. K., Korfiatis, N., Sivakumar, V. J., y Lytras, M. D. (2018). Mobile shopping apps adoption and perceived risks: A cross-country perspective utilizing the Unified Theory of Acceptance and use of technology. Computers in Human Behavior, 86, 109-128. DOI: https://doi.org/10.1016/j.chb.2018.04.017

Costa, P. T., y McCrae, R. R. (1992). Revised NEO personality inventory (NEO PI-R) and NEO five-factor inventory (NEO-FFI): Professional manual. Odessa, FL: Psychological Assessment Resources.

Cox, J. (2004). Ubiquitous consumption and the marketing mix. Journal of Internet Commerce, 3(2), 21-32. DOI: https://doi.org/10.1300/J179v03n02 02

Cudeck, R., y Browne, M. W. (1993). Alternative ways of assessing model fit. En K. A. Bollen y J. S. Long (Eds.), Testing structural equation models (pp. 1-9). Newbury Park, CA: Sage.

Davis, F. D. (1989). Perceived usefulness, perceived ease of use, and user acceptance of information technology. MIS Quarterly, 13(3), 319-340. DOI: https://doi.org/10.2307/249008

Dawson, J. F. (2014). Moderation in management research: What, why, when, and how. Journal of Business and Psychology, 29(1), 1-19. DOI: https://doi.org/10.1007/s10869-013-9308-7

Dickerson, M. D., y Gentry, J. W. (1983). Characteristics of adopters and non-adopters of home computers. Journal of Consumer research, 10(2), 225-235. DOI: https://doi.org/10.1086/208961

Dickman, S. J. (1990). Functional and dysfunctional impulsivity: Personality and cognitive correlates. Journal of Personality and Social Psychology, 58(1), 95-102. DOI: https://doi.org/10.1037/0022-3514.58.1.95

Edwards, J. R., y Lambert, L. S. (2007). Methods for integrating moderation and mediation: A general analytical framework using moderated path analysis. Psychological Methods, 12(1), 1-22.

DOI: https://doi.org/10.1037/1082-989X.12.1.1

Faqih, K. M. S., y Jaradat, M.-I. R. M. (2015). Assessing the moderating effect of gender differences and individualism-collectivism at individual-level on the adoption of mobile commerce technology: TAM3 perspective. Journal of Retailing and Consumer Services, 22, 37-52.

DOI: https://doi.org/10.1016/j.jretconser.2014.09.006 
Fishbein, M. A., y Ajzen, I., (1975). Belief, attitude, intention, and behavior: An introduction to theory and research. Reading, MA: Addison-Wesley.

Fornell, C., y Larcker, D. F. (1981). Evaluating structural equation models with unobservable variables and measurement error. Journal of Marketing Research, 18(1), 39-50. DOI: https://doi.org/10.1177/002224378101800104

Groß, M. (2015). Exploring the acceptance of technology for mobile shopping: An empirical investigation among smartphone users. The International Review of Retail, Distribution and Consumer Research, 25(3), $215-235$. DOI: https://doi.org/10.1080/09593969.2014.988280

Gupta, A., y Arora, N. (2017). Understanding determinants and barriers of mobile shopping adoption using behavioral reasoning theory. Journal of Retailing and Consumer Services, 36, 1-7. DOI: https://doi.org/10.1016/j.jretconser.2016.12.012

Hair, J. F., Anderson, R. E., Tatham, R. L., y Black, W. C. (1999). Análisis multivariante. Madrid: Prentice Hall.

Hair, J. F., Anderson, R. E., Tatham, R. L., y Black, W. C. (2010). Multivariate data analysis: A global perspective. (7th ed.). Upper Saddle River, NJ: Pearson Prentice-Hall.

Hernandez, B., Jimenez, J., y Martin, M. (2009). The impact of self-efficacy, ease of use and usefulness on e-purchasing: An analysis of experienced e-shoppers. Interacting with Computers, 21(1-2), 146-156. DOI: https://doi.org/10.1016/j.intcom.2008.11.001

Hirschman, E. C. (1980). Innovativeness, novelty seeking, and consumer creativity. Journal of Consumer Research, 7(3), 283-295. DOI: https://doi.org/10.1086/208816

Hu, L.-T., y Bentler, P. M. (1999). Cutoff criteria for fit indexes in covariance structure analysis: Conventional criteria versus new alternatives. Structural Equation Modeling: a Multidisciplinary Journal, 6(1), 1-55. DOI: https://doi.org/10.1080/10705519909540118

Huang, J., y Zhou, L. (2018). Timing of web personalization in mobile shopping: A perspective from uses and gratifications theory. Computers in Human Behavior, 88, 103-113. DOI: https://doi.org/10.1016/j.chb.2018.06.035

Igbaria, M. (1992). An examination of microcomputer usage in Taiwan. Information \& Management, 22(1), $19-28$. DOI: https://doi.org/10.1016/0378-7206(92)90003-X

Igbaria, M., y Tan, M. (1997). The consequences of information technology acceptance on subsequent individual performance. Information \& Management, 32(3), 113-121. DOI: https://doi.org/10.1016/S0378-7206(97)00006-2

Igbaria, M., y Toraskar, K. (1992). Impact of end user computing on the individual: An integrated model. Information Technology \& People, 6(4), 271-292. DOI: https://doi.org/10.1108/09593849310076286

Igbaria, M., Guimaraes, T., y Davis, G. B. (1995). Testing the determinants of microcomputer usage via a structural equation model. Journal of Management Information Systems, 11(4), 87-114. DOI: https://doi.org/10.1080/07421222.1995.11518061

Jiménez, N., San-Martín, S., y Puente, N. (2019). The path to mobile shopping compatibility. The Journal of High Technology Management Research, 30(1), 15-26. DOI: https://doi.org/10.1016/j.hitech.2018.12.006

Kacen, J. J., y Lee, J. A. (2002). The influence of culture on consumer impulsive buying behavior. Journal of Consumer Psychology, 12(2), 163-176. DOI: https://doi.org/10.1207/S15327663JCP1202 08

Kamal, S., Chu, S. C., y Pedram, M. (2013). Materialism, attitudes, and social media usage and their impact on purchase intention of luxury fashion goods among American and Arab young generations. Journal of Interactive Advertising, 13(1), 27-40. DOI: https://doi.org/10.1080/15252019.2013.768052

Kapoor, A. P., y Vij, M. (2018). Technology at the dinner table: Ordering food online through mobile apps. Journal of Retailing and Consumer Services, 43, 342-351. DOI: https://doi.org/10.1016/j.jretconser.2018.04.001

Kats, R. (19 de febrero de 2020). AliExpress and Amazon are bolstering Spain's booming ecommerce market. New York, NY: eMarketer. Recuperado de: https://www.emarketer.com/content/aliexpress-and-amazon-arebolstering-spain-s-booming-ecommerce-market

Kenny, D. A., Kaniskan, B., y McCoach, D. B. (2015). The performance of RMSEA in models with small degrees of freedom. Sociological Methods \& Research, 44(3), 486-507. DOI: https://doi.org/10.1177/0049124114543236

Khan, A. N., Cao, X., y Pitafi, A. H. (2019). Personality traits as predictor of M-payment systems: A SEM-neural networks approach. Journal of Organizational and End User Computing (JOEUC), 31(4), 89-110. DOI: https://doi.org/10.4018/JOEUC.2019100105

Khorrami, M. S., Esfidani, M. R., y Delavari, S. (2015). The effect of situational factors on impulse buying and compulsive buying: Clothing. International Journal of Management, Accounting and Economics, 2(8), 823-837. 
Kim, C., Li, W., y Kim, D. J. (2015). An empirical analysis of factors influencing M-shopping use. International Journal of Human-Computer Interaction, 31(12), 974-994.

DOI: https://doi.org/10.1080/10447318.2015.1085717

Kim, M., Kim, J., Choi, J., y Trivedi, M. (2017). Mobile shopping through applications: Understanding application possession and mobile purchase. Journal of Interactive Marketing, 39, 55-68.

DOI: https://doi.org/10.1016/j.intmar.2017.02.001

Kim, N. Y. (2018). The effect of advertising content control on advertising effectiveness in the different forced exposure circumstance. Journal of Promotion Management, 24(6), 845-862. DOI: https://doi.org/10.1080/10496491.2017.1408528

Lee, H. (2018). Intrinsic and extrinsic motivations affecting impulse-buying tendency in mobile shopping. Social Behavior and Personality: An International Journal, 46(4), 683-694. DOI: https://doi.org/10.2224/sbp.6693

Lee, T., Park, C., y Jun, J. (2014). Two faces of mobile shopping: Self-efficacy and impulsivity. International Journal of E-Business Research (IJEBR), 10(1), 15-32. DOI: https://doi.org/10.4018/ijebr.2014010102

Lejoyeux, M., Mathieu, K., Embouazza, H., Huet, F., y Lequen, V. (2007). Prevalence of compulsive buying among customers of a Parisian general store. Comprehensive Psychiatry, 48(1), 42-46. DOI: https://doi.org/10.1016/j.comppsych.2006.05.005

Li, M., Huang, L., Tan, C. H., y Wei, K. K. (2013). Helpfulness of online product reviews as seen by consumers: Source and content features. International Journal of Electronic Commerce, 17(4), 101-136. DOI: https://doi.org/10.2753/JEC1086-4415170404

Lim, S. H., Lee, S., y Kim, D. J. (2017). Is online consumers' impulsive buying beneficial for e-commerce companies? An empirical investigation of online consumers' past impulsive buying behaviors. Information Systems Management, 34(1), 85-100. DOI: https://doi.org/10.1080/10580530.2017.1254458

Lissitsa, S., y Kol, O. (2019). Four generational cohorts and hedonic m-shopping: Association between personality traits and purchase intention. Electronic Commerce Research, 1-26. DOI: https://doi.org/10.1007/s10660-019-09381-4

Liu, S., y Xiao, L. (2018). Research on the influence of website characteristics on consumers' impulsive purchase intention. En Proceedings of the 2018 2nd International Conference on Education, Economics and Management Research (ICEEMR 2018). Singapore (Singapore), June 9-10, 2018. Atlantis Press. DOI: https://doi.org/10.2991/iceemr-18.2018.176

Lu, J., Yao, J. E., y Yu, C.-S. (2005). Personal innovativeness, social influences and adoption of wireless Internet services via mobile technology. The Journal of Strategic Information Systems, 14(3), 245-268. DOI: https://doi.org/10.1016/j.jsis.2005.07.003

Luarn, P., y Lin, H.-H. (2005). Toward an understanding of the behavioral intention to use mobile banking. Computers in Human Behavior, 21(6), 873-891. DOI: https://doi.org/10.1016/j.chb.2004.03.003

Mahatanankoon, P. (2007). The effects of personality traits and optimum stimulation level on text-messaging activities and m-commerce intention. International Journal of Electronic Commerce, 12(1), 7-30. DOI: https://doi.org/10.2753/JEC1086-4415120101

McDonald, R. P., y Ho, M.-H. R. (2002). Principles and practice in reporting structural equation analyses. Psychological Methods, 7(1), 64-82. DOI: https://doi.org/10.1037/1082-989X.7.1.64

Miao, M., Jalees, T., Qabool, S., y Zaman, S. I. (2019). The effects of personality, culture and store stimuli on impulsive buying behavior. Asia Pacific Journal of Marketing and Logistics, 32(1), 188-204. DOI: https://doi.org/10.1108/APIML-09-2018-0377

Millar, M., y Thomas, R. (2009). Discretionary activity and happiness: The role of materialism. Journal of Research in Personality, 43(4), 699-702. DOI: https://doi.org/10.1016/i.jp.2009.03.012

Mowen, J. C. (2000). The 3M model of motivation and personality: Theory and empirical applications to consumer behavior. Norwell, MA: Kluwer Academic Press. DOI: https://doi.org/10.1007/978-1-4757-6708-7

Natarajan, T., Balasubramanian, S. A., y Kasilingam, D. L. (2017). Understanding the intention to use mobile shopping applications and its influence on price sensitivity. Journal of Retailing and Consumer Services, 37, 8-22. DOI: https://doi.org/10.1016/i.jretconser.2017.02.010

Natarajan, T., Balasubramanian, S. A., y Kasilingam, D. L. (2018). The moderating role of device type and age of users on the intention to use mobile shopping applications. Technology in Society, 53, 79-90. DOI: https://doi.org/10.1016/j.techsoc.2018.01.003

Nicholls, J. (1984). Conceptions of ability and achievement motivation. En R. Ames y C. Ames (Eds.), Research on motivation in education (pp. 39-73). New York, NY: Academic Press.

Nicholls, J. (1989). The competitive ethos and democratic education. Cambridge, MA: Harvard University Press. 
Observatorio Nacional de las Telecomunicaciones y de la Sociedad de la Información. (2019). El comercio electrónico B2C en España. 2018. Madrid: ONTSI. DOI: http://doi.org/10.30923/2172-458x/b2c/201911

O'Guinn, T. C., y Faber, R. J. (1989). Compulsive buying: A phenomenological exploration. Journal of Consumer Research, 16(2), 147-157. DOI: https://doi.org/10.1086/209204

Okazaki, S., y Mendez, F. (2013). Exploring convenience in mobile commerce: Moderating effects of gender. Computers in Human Behavior, 29(3), 1234-1242. DOI: https://doi.org/10.1016/j.chb.2012.10.019

Otero-López, J. M., y Villardefrancos, E. (2013). Five-Factor Model personality traits, materialism, and excessive buying: A mediational analysis. Personality and Individual Differences, 54(6), 767-772. DOI: https://doi.org/10.1016/i.paid.2012.12.013

Parsad, C., Prashar, S., y Tata, V. S. (2019). Influence of personality traits and social conformity on impulsive buying tendency: Empirical study using 3M model. International Journal of Strategic Decision Sciences (IJSDS), 10(2), 107-124. DOI: https://doi.org/10.4018/IJSDS.2019040106

Parsad, C., Prashar, S., Vijay, T. S., y Kumar, M. (2018). In-store stimuli and impulsive buying behaviour: Modeling through regression equation. International Journal of Strategic Decision Sciences (IJSDS), 9(3), 95-112. DOI: https://doi.org/10.4018/IJSDS.2018070105

Patel, V., Das, K., Chatterjee, R., y Shukla, Y. (2020). Does the interface quality of mobile shopping apps affect purchase intention? An empirical study. Australasian Marketing Journal (AMJ). DOI: https://doi.org/10.1016/j.ausmj.2020.08.004

Peck, J., y Childers, T. L. (2006). If I touch it I have to have it: Individual and environmental influences on impulse purchasing. Journal of Business Research, 59(6), 765-769. DOI: https://doi.org/10.1016/i.jbusres.2006.01.014

Podoshen, J. S., y Andrzejewski, S. A. (2012). An examination of the relationships between materialism, conspicuous consumption, impulse buying, and brand loyalty. Journal of Marketing Theory and Practice, 20(3), 319-334. DOI: https://doi.org/10.2753/MTP1069-6679200306

Preacher, K. J., Rucker, D. D., y Hayes, A. F. (2007). Addressing moderated mediation hypotheses: Theory, methods, and prescriptions. Multivariate Behavioral Research, 42(1), 185-227. DOI: https://doi.org/10.1080/00273170701341316

Putrawan, I. M. (2020). Personality on green consumer behaviour. International Journal of Psychosocial Rehabilitation, 24(2), 2374-2379. DOI: https://doi.org/10.37200/IJPR/V24I2/PR200534

Richins, M. L. (2013). When wanting is better than having: Materialism, transformation expectations, and product-evoked emotions in the purchase process. Journal of Consumer Research, 40(1), 1-18. DOI: https://doi.org/10.1086/669256

Roger, E. M. (1995). Diffusion of innovation. (4th ed.). New York, NY: The Freeman Press.

Rook, D. W. (1987). The buying impulse. Journal of Consumer Research, 14(2), 189-199. DOI: https://doi.org/10.1086/209105

Rook, D. W., y Fisher, R. J. (1995). Normative influences on impulsive buying behavior. Journal of Consumer Research, 22(3), 305-313. DOI: https://doi.org/10.1086/209452

Rose, P. (2007). Mediators of the association between narcissism and compulsive buying: The roles of materialism and impulse control. Psychology of Addictive Behaviors, 21(4), 576-581. DOI: https://doi.org/10.1037/0893-164X.21.4.576

Sarrazin, P., Roberts, G., Cury, F., Biddle, S., y Famose, J. P. (2002). Exerted effort and performance in climbing among boys: The influence of achievement goals, perceived ability, and task difficulty. Research Quarterly for Exercise and Sport, 73(4), 425-436. DOI: https://doi.org/10.1080/02701367.2002.10609042

Seegers, G., van Putten, C. M., y De Brabander, C. J. (2002). Goal orientation, perceived task outcome and task demands in mathematics tasks: Effects on students' attitude in actual task settings. British Journal of Educational Psychology, 72(3), 365-384. DOI: https://doi.org/10.1348/000709902320634366

Šeinauskienè, B., Maščinskienè, J., Petrike, I., y Rutelionè, A. (2016). Materialism as the mediator of the association between subjective well-being and impulsive buying tendency. Inžineriné Ekonomika, 27(5), 594-606. DOI: https://doi.org/10.5755/j01.ee.27.5.13830

Shahjehan, A., Zeb, F., y Saifullah, K. (2012). The effect of personality on impulsive and compulsive buying behaviors. African Journal of Business Management, 6(6), 2187-2194. Recuperado de: https://pdfs.semanticscholar.org/7860/88073ed2193b2138c3f64fd2d170ae4fc36d.pdf

Silvera, D. H., Lavack, A. M., y Kropp, F. (2008). Impulse buying: The role of affect, social influence, and subjective wellbeing. Journal of Consumer Marketing, 25(1), 23-33. DOI: https://doi.org/10.1108/07363760810845381

Soutter, A. R. B., Bates, T. C., y Mõttus, R. (2020). Big five and HEXACO personality traits, proenvironmental attitudes, and behaviors: A meta-analysis. Perspectives on Psychological Science, 15(4), 913-941. DOI: https://doi.org/10.1177/1745691620903019 
Spence, J. T., y Helmreich, R. L. (1983). Achievement-related motives and behaviors. En J. T. Spence (Ed.), Achievement and achievement motives: Psychological and sociological approaches (pp. 10-74). San Francisco, CA: Freeman.

Statista (2020). Porcentaje de usuarios que realizaron compras a través de dispositivos móviles en España entre 2014 y 2018. Recuperado de: https://es.statista.com/estadisticas/872927/porcentaje-de-usuarios-querealizaron-compras-por-dispositivos-moviles/

Stefańska, M., y Śmigielska, G. (2020). Impulse purchase in virtual environment and price sensitivity of young consumers: Results of empirical research. Economy \& Market Communication Review/Casopis za Ekonomiju $i$ Trzisne Komunikacije, 10(1).

Steiger, J. H., y Lind, J. C. (1980). Statistically-based tests for the number of common factors. En The Meeting of the Psychometric Society. Iowa City, IA.

Straub, D., Limayem, M., y Karahanna-Evaristo, E. (1995). Measuring system usage: Implications for IS theory testing. Management Science, 41(8), 1328-1342. DOI: https://doi.org/10.1287/mnsc.41.8.1328

Sun, G., Wang, W., Cheng, Z., Li, J., y Chen, J. (2017). The intermediate linkage between materialism and luxury consumption: Evidence from the emerging market of China. Social Indicators Research, 132, 475-487. DOI: https://doi.org/10.1007/s11205-016-1273-X

Sun, T., y Wu, G. (2011). Trait predictors of online impulsive buying tendency: A hierarchical approach. Journal of Marketing Theory and Practice, 19(3), 337-346. DOI: https://doi.org/10.2753/MTP1069-6679190307

Tenenbaum, G., Hall, H. K., Calcagnini, N., Lange, R., Freeman, G., y Lloyd, M. (2001). Coping with physical exertion and negative feedback under competitive and self-standard conditions. Journal of Applied Social Psychology, 31(8), 1582-1626. DOI: https://doi.org/10.1111/j.1559-1816.2001.tb02743.x

Thompson, R. L., Higgins, C. A., y Howell, J. M. (1991). Personal computing: Toward a conceptual model of utilization. MIS Quarterly, 15(1), 125-143. DOI: https://doi.org/10.2307/249443

Turkyilmaz, C. A., Erdem, S., y Uslu, A. (2015). The effects of personality traits and website quality on online impulse buying. Procedia-Social and Behavioral Sciences, 175, 98-105. DOI: https://doi.org/10.1016/j.sbspro.2015.01.1179

Vahdat, A., Alizadeh, A., Quach, S., y Hamelin, N. (2020). Would you like to shop via mobile app technology? The technology acceptance model, social factors and purchase intention. Australasian Marketing Journal (AMJ). DOI: https://doi.org/10.1016/j.ausmj.2020.01.002

Venkatesh, V., y Bala, H. (2008). Technology acceptance model 3 and a research agenda on interventions. Decision Sciences, 39(2), 273-315. DOI: https://doi.org/10.1111/j.1540-5915.2008.00192.x

Venkatesh, V., Thong, J. Y., y Xu, X. (2012). Consumer acceptance and use of information technology: Extending the unified theory of acceptance and use of technology. MIS Quarterly, 36(1), 157-178. DOI: https://doi.org/10.2307/41410412

Verplanken, B., y Herabadi, A. (2001). Individual differences in impulse buying tendency: Feeling and no thinking. European Journal of Personality, 15(S1), S71-S83. DOI: https://doi.org/10.1002/per.423

Verplanken, B., Herabadi, A. G., Perry, J. A., y Silvera, D. H. (2005). Consumer style and health: The role of impulsive buying in unhealthy eating. Psychology \& Health, 20(4), 429-441.

DOI: https://doi.org/10.1080/08870440412331337084

Wells, J. D., Parboteeah, V., y Valacich, J. S. (2011). Online impulse buying: Understanding the interplay between consumer impulsiveness and website quality. Journal of the Association for Information Systems, 12(1), 32-56. DOI: https://doi.org/10.17705/1jais.00254

Wills, T. A., Sandy, J. M., y Yaeger, A. (2000). Temperament and adolescent substance use: An epigenetic approach to risk and protection. Journal of Personality, 68(6), 1127-1151. DOI: https://doi.org/10.1111/1467-6494.00129

Wixom, B. H., y Todd, P. A. (2005). A theoretical integration of user satisfaction and technology acceptance. Information Systems Research, 16(1), 85-102. D0I: https://doi.org/10.1287/isre.1050.0042

Wong, Y.-T., Osman, S., Jamaluddin, A., y Yin-Fah, B. C. (2012). Shopping motives, store attributes and shopping enjoyment among Malaysian youth. Journal of Retailing and Consumer Services, 19(2), 240-248. DOI: https://doi.org/10.1016/i.jretconser.2012.01.005

Wu, J.-H., y Wang, S.-C. (2005). What drives mobile commerce?: An empirical evaluation of the revised technology acceptance model. Information \& Management, 42(5), 719-729. DOI: https://doi.org/10.1016/j.im.2004.07.001

Wu, W., Wang, H., Lee, H.-Y., Lin, Y.-T., y Guo, F. (2019). How machiavellianism, psychopathy, and narcissism affect sustainable entrepreneurial orientation: The moderating effect of psychological resilience. Frontiers in psychology, 10, 779. DOI: https://doi.org/10.3389/fpsyg.2019.00779 
Xiao, S. H., y Nicholson, M. (2013). A multidisciplinary cognitive behavioural framework of impulse buying: A systematic review of the literature. International Journal of Management Reviews, 15(3), 333-356. DOI: https://doi.org/10.1111/j.1468-2370.2012.00345.x

Yang, K., y Forney, J. C. (2013). The moderating role of consumer technology anxiety in mobile shopping adoption: Differential effects of facilitating conditions and social influences. Journal of Electronic Commerce Research, 14(4), 334-347. Recuperado de: http://www.jecr.org/sites/default/files/14_4_p04.pdf

Yazdanparas, A., y Alhenawi, Y. (2017). Personality and borrowing behavior: An examination of the role of need for material resources and need for arousal traits on household's borrowing decisions. Financial Services Review, 26(1), 55-85.

Zhang, L., Zhu, J., y Liu, Q. (2012). A meta-analysis of mobile commerce adoption and the moderating effect of culture. Computers in Human Behavior, 28(5), 1902-1911. DOI: https://doi.org/10.1016/j.chb.2012.05.008

Zhang, X., Prybutok, V. R., y Strutton, D. (2007). Modeling influences on impulse purchasing behaviors during online marketing transactions. Journal of Marketing Theory and Practice, 15(1), 79-89. DOI: https://doi.org/10.2753/MTP1069-6679150106

Zheng, X., Men, J., Yang, F., y Gong, X. (2019). Understanding impulse buying in mobile commerce: An investigation into hedonic and utilitarian browsing. International Journal of Information Management, 48, 151-160. DOI: https://doi.org/10.1016/j.ijinfomgt.2019.02.010

Zhou, T., y Lu, Y. (2011). The effects of personality traits on user acceptance of mobile commerce. International Journal of Human-Computer Interaction, 27(6), 545-561. DOI: https://doi.org/10.1080/10447318.2011.555298

Ziano, I., y Villanova, D. (2019). You'd use it more than me: Overestimating products' usefulness to others because of self-serving materialism attributions. PsyArXiv. DOI: https://doi.org/10.31234/osf.io/938m7 